RESEARCH IN BRIEF

\title{
THE CONTRIBUTION OF COMMUNITY PARENT ADVISORS TO IMPROVING HEALTH IN SCHOOL COMMUNITIES IN A PRIMARY CARE TRUST IN NORTHERN ENGLAND
}

\section{AIMS}

This article reports on a preliminary study that looked at the introduction of a new role, the Community Parent Advisor (CPA), to assist school nurses in health education and promotion. The study aimed firstly, to describe and consider the first year of the role and secondly, to look for health improvement in the school community from the perspective of the CPAs and school nurses. During the literature review it became apparent that research into school nursing and allied roles was limited, with little available evidence of effectiveness (Wainwright et al. 2000, Bunn 2005 in Christian 2005, p. 12). Occasionally allusion was made to elements of the school nurses' role being undertaken by assistive personnel to make the most effective use of resources (Allensworth 1996, p. 285).

\section{BACKGROUND}

The study took place in a Primary Care Trust in Northern England where twenty CPAs were placed in primary schools in areas of high deprivation and poor health (Eynon and Reilly 2004). Six months before their appointment, Community Nursing had been re-organised into locality teams, which the CPAs joined. CPAs were overseen by a school nurse in carrying out their job description which stated that they were to provide assistance to the whole school community in accordance with the Public Health Agenda ... identify the health promotion needs and deliver health education advice to parents and children within a defined school.'

\section{METHODS}

A qualitative approach was taken to elicit the CPAs' and school nurses' perspective of the role. Semi-structured interviews were conducted with seven out of the fourteen CPAs remaining in post and three of the five school nurses who worked directly with them. The interviews were taped, transcribed verbatim and subjected to content analysis (Strauss and Corbin 1998). Prior to data collection ethical approval was given by the University Research Ethics Committee, regional Research and Governance Unit and Local Research Ethics Committee.

\section{Sample}

A purposeful sample was selected according to convenience and within time limitations. All CPAs and school nurses who worked with them were approached. All who responded were included in the study.

Data analysis involved increasingly focused levels of data coding until the 'storyline' emerged (Strauss and Corbin 1998, p. 148). The credibility of the study was enhanced through member-checking, audit trail and peer debriefing. The data itself allowed for triangulation of information for accuracy. 


\section{FINDINGS}

The findings were in two parts: i) a description of the role with elements of its effectiveness and ii) identification of three main themes surrounding the introduction of the role. With regard to the first, the role was described as providing health advice and support for parents, children and school staff. CPAs acted as role models for school parents, especially as they came from local communities. They were able to provide additional teaching on health promotion and education for children's classes and co-ordinate some parents' classes. The CPA provided a link to other sources of health advice and outside agencies e.g. healthy lifestyle workers and adult education. They were able to co-ordinate lengthy health events in school that would otherwise not have happened. CPAs also assisted school nurses in various ways, including with routine screening and immunisations and some work in secondary schools.

Data analysis showed that the main health promotion needs identified in the primary schools were head lice control, healthy eating, dental health, hygiene and self-care and information for parents. Health promotion methods that were most effective were: ready access to advice and support, tailoring assistance to the school community, reinforcement of healthy messages and encouraging community participation in identifying and meeting the needs.

The findings demonstrated that the CPA role had an impact on children, families, the school nursing service and school staff. They raised children's awareness of healthy lifestyle choices, increased healthy food intake at events and children took healthy messages home. Through dealing with head lice and hygiene, children's self-care skills and self-esteem improved and school days were no longer lost due to lice treatment. Families' knowledge and skills around healthy living were reported to have increased. School nurse time was released by CPAs dealing with head lice and helping with immunisations and screening but their time was taken up with training and managing the CPAs. Overall school nurses said the amount of health promotion available through schools was increased. As one CPA said, 'It's marvellous to know that you're making a difference'.

The second part of the findings identified three main themes derived from the data: 1) clarity of communication 2) quality of relationships and 3) individual contribution by the CPA. The outcome of the role rested on these themes. The study found that there was a lack of clear communication between senior management and school nurses. School nurses, who were expected to manage the CPAs on a daily basis, did not own the vision for the new role and instead felt ill-prepared and imposed upon, as one school nurse said, 'The second wave of community parents started in the November, by which time there'd been an outcry'. This damaged relationships between school nurses and CPAs although some relationships improved with time and effort.

CPAs generally communicated well with the school community, although this depended upon first building up trust and breaking down any barriers. Communication and the quality of relationships were closely linked; for example healthy messages could only be delivered after positive relationships 
were built. The character of the CPA affected relationships with their school nurse and school. Those who were confident, outgoing, persevering, adaptable, prepared to take the initiative and be creative were more likely to succeed in building good, working relationships.

\section{Limitations}

The study was limited by the sample in that it only included those willing and able to discuss the role and who remained in post.

\section{CONCLUSIONS}

The participants took the view that the introduction of the CPA role brought health promotion benefits to the school communities. Although the way the change was managed reduced the role's effectiveness, by creating a poor foundation on which to build, the role nevertheless has potential to effectively deliver the public health agenda.

\section{RELEVANCE TO CLINICAL PRACTICE}

The findings of this study hold utility for application of a similar role within other Primary Care Trusts to promote public health, although the change needs to be well planned and carefully managed.

\section{ACKNOWLEDGEMENTS}

No external funding was sought for this study however I would like to thank my academic supervisor and the director of the primary care trust for their support. 